\title{
Reflexión. Mediación: Toma de Decisiones ante Cambios Familiares
}

\section{Reflection. Mediation: Decision Making in Family Changes}

\author{
Beatriz G. Saguar y Guadalupe Viola Nevado \\ Centro de Apoyo a las Familias, Madrid, España
}

\begin{abstract}
Resumen. El artículo plantea un modelo de mediación surgido de la práctica en un recurso público, que integra aspectos nacidos de las necesidades expresadas por las familias y observadas por las mediadoras, no considerados normalmente como parte del proceso "clásico" de lo que se entiende por mediación y que, sin embargo, dan resultados en la práctica. Es el modelo de mediación como acompañamiento en procesos de toma de decisiones ante cambios familiares.

Por otra parte el artículo hace referencia a la necesidad de una redefinición de la mediación en el contexto profesional en el que está inscrito, llevada a cabo por las propios mediadores, como la única forma de "definir" de manera clara el trabajo que realmente se lleva a cabo y facilitar, de este modo, la coordinación, interacción y comunicación con otros actores que forman parte del espacio de ayuda familiar.

Palabras clave: acompañamiento, cambio familiar, coordinación, coparentalidad, mediación, toma de decisiones.
\end{abstract}

\begin{abstract}
The article presents a model of mediation practice emerged from a public resource that integrates the needs expressed by participants and observed by the mediators, not normally considered as part of the "classic" mediation model and that, however, yield results in practice. It is the mediation model as accompanying decision-making processes to family changes.

Moreover, the article refers to the need for a redefinition of mediation in the professional context in which it is registered, carried out by the mediators themselves, as the only way to "define" clearly the work actually performed and provided, thus, coordination, interaction and communication with other actors who are part of the area of family support.

Keywords: accompanying, coordination, coparents, decision-making, family change, mediation.
\end{abstract}

\section{Lo que otros dicen que es mediación}

Tal y como algunos autores recogen, el concepto de mediación ha ido evolucionando a medida que también lo hacían su práctica y sus ámbitos de actuación, en función de los modelos teóricos que se iban desarrollando (Bolaños 2008).

Alguna de estas definiciones más clásicas de mediación hacen referencia a que se trata de un proce-

La correspondencia sobre este artículo debe enviarse a la primera autora al Centro de Apoyo a las Familias 4. c/ Rios Rosas, 39, 2 ext derecha. 28003 Madrid. E-mail: beatrizsaguar@hotmail.com so, un sistema, una solución a conflictos, un recurso, una técnica, por ejemplo, Haynes (1993), define "Mediación como un proceso en virtud del cual un tercero, el mediador, ayuda a los participantes en una situación conflictiva a su resolución, que se expresa en un acuerdo".

Carmen Maganto Mateo (2004) plantea la mediación como "un sistema para llegar a un acuerdo vía negocial, cuya tramitación puede ser conducida por el juez interviniente, por algún funcionario judicial o por otro profesional capacitado para desempeñar dicha tarea"... 
Trinidad Bernal (2002) habla de "mediación, una solución a los conflictos de pareja". Y añade que la mediación es "una técnica pacífica de resolver conflictos donde el protagonismo lo tienen las partes, cambiando el rol de los actores intervinientes en la situación conflictiva".

Por otro lado, la definición de la exposición de motivos de la ley 15/2005 de 8 de julio que modifica al CC y a la LEC. Se refiere a la "Mediación como recurso voluntario alternativo de solución de los litigios familiares por vía de mutuo acuerdo con la intervención de un mediador, imparcial y neutral".

Sin embargo nuestro punto de partida es pensar en la práctica de la mediación familiar, como el motor que hace evolucionar el modelo de mediación, más allá de los modelos teóricos propuestos a priori, es decir, partimos de la práctica, para después generar una propuesta de modelo teórico.

Entendemos evolución, en este sentido, como un proceso que trasciende y completa el concepto más clásico de mediación familiar, definido como "método de resolución de conflictos aplicado a casos concretos".

En nuestro caso, abordamos el planteamiento de la mediación familiar desde su práctica en el contexto de un centro público, dependiente del Ayuntamiento de Madrid, de acceso directo y situado en un barrio densamente poblado del sureste de Madrid.

En ese espacio de atención directa, a un sinnúmero de familias con demandas absolutamente variadas, la mediación familiar sólo puede plantearse como una herramienta flexible. Una herramienta que pueda adaptarse y ofrecer la posibilidad a las familias de retomar los aspectos de su vida que les preocupan. Y todo esto, con objetivos que pueden ser absolutamente variados, que surgen sobre la marcha de sus necesidades y que van desde la resolución, la organización, la mejora de la comunicación o la capacidad de compartir un mismo espacio. En resumen, la toma de decisiones en situaciones de cambio.

Ofrecer a las personas la capacidad de retomar aspectos puntuales de su vida que les preocupan, supone para nosotras mediadoras, favorecer un espacio en el que redescubran sobre todo, su capaci- dad de actuación, de reflexión y de toma de decisiones. En este sentido, nuestro papel consiste en acompañar a las familias a lo largo del proceso, desde la toma de conciencia de sus dificultades y la escucha activa de sus necesidades, hasta la consecución de decisiones que faciliten el cambio familiar.

\section{Lo que las mediadoras y las familias hacemos en mediación}

Nos gustaría empezar este apartado, definiendo la mediación no tanto como un método de trabajo, sino como un espacio que se construye con las aportaciones no sólo del mediador sino también de las familias. Se trata de un espacio facilitador de toma de decisiones en situaciones de cambio familiar tales como el divorcio. Nuestro objetivo en este apartado es mostrar cómo la actuación del mediador, los objetivos de la mediación y el espacio de mediación se transforman, adaptan y flexibilizan, en función de las necesidades de las familias y del tipo de dificultad que presenten. Es por ello, que, para nosotras, la mediación se convierte muchas veces en un espacio terapéutico de acompañamiento a las familias. $¿$ Quiere esto decir que trabajamos en un contexto de terapia familiar? No, nosotras lo entendemos como trabajar en mediación dentro de un contexto terapéutico. No entendemos otra forma de trabajar con familias en proceso de cambio, que no sea atendiendo tanto a sus demandas más prácticas, como a sus necesidades emocionales. Esta forma de intervenir con las familias, es transversal, sea cual sea la necesidad que la familia presenta.

Hemos querido centrarnos en este artículo en el trabajo que hacemos con familias que están inmersas en un procedimiento de separación y/o divorcio (casados o no). Y en nuestro punto de partida, no podemos perder de vista a los menores, que, sin tener la oportunidad de protegerse a si mismos, se ven inmersos en un conflicto de preocupantes consecuencias para ellos/ellas. No tenemos ninguna duda de que las grandes víctimas en un divorcio (y muchas veces olvidadas tanto por las instituciones como por los profesionales que intervenimos con las familias) son los menores. Las pérdidas son cuantiosas a todos los niveles. Para un niño o niña, renun- 
ciar a volver a ver juntos a sus padres, es, sin duda una de las mayores pérdidas de su vida. Y como toda pérdida, implica la elaboración de un duelo, que será infinitamente más llevadero si lo hacen con la ayuda de sus padres.

A lo largo de estos años de trabajo con familias, hemos observado como el impacto del divorcio en los menores es proporcional al nivel de conflictividad con que los padres manejen dicho divorcio. No conocemos a ningún niño o niña que a priori se muestre contento/a ante la noticia de que sus padres se separan. Sin embargo creemos que quien mejor puede amortiguar el impacto de esta pérdida son los propios padres con un manejo adecuado de la situación.

Nos encontramos demasiado a menudo con padres y madres dispuestos a llevar a sus hijos e hijas a los mejores psicólogos de la ciudad para que les "ayuden a superarlo". Y nuestro mensaje en este sentido es siempre el mismo: vosotros sois las personas más adecuadas para ayudar a vuestros hijos e hijas a pasarlo lo mejor posible. En la medida que los padres se comunican, se escuchan, miran a sus hijos/as y deciden, los menores se adaptarán a los cambios con menos resistencias. Si los menores reciben información sobre los cambios ${ }^{1}$ que implica la ruptura, podrán buscar herramientas que les permitan enfrentarse a dichos cambios; pero si los menores no son informados de lo que está ocurriendo entre sus padres, difícilmente sabrán qué hacer.

En este sentido, entendemos el espacio de mediación como un espacio desde el que promover la coparentalidad. Entendemos por coparentalidad, reforzar el "equipo de padres" y entender que aunque la relación de pareja termina, la relación de padres debe fortalecerse en beneficio de los hijos/as.

Por todo lo anterior, la intervención del mediador configura un nuevo espacio, en el que acompañar a familias que pasan por las situaciones anteriormente descritas, y en este sentido, a lo largo de estos años de trabajo nos hemos encontrado con familias con

La información que se debe dar a los menores en situaciones de divorcio o ruptura, daría para escribir largos textos, pero queremos señalar la importancia que tiene que los menores sepan lo que está pasando con información adaptada a su edad y sin entrar en cuestiones que tienen que ver con la relación de pareja, sobre todo los cambios que más les afectan (cambio de domicilio, cambio de colegio). necesidades y en situaciones muy diferentes que hemos considerado agrupar de esta manera:

\section{¿Nos separamos o no?}

Si nos remitimos estrictamente a la teoría sobre mediación, seguramente encontremos mucha documentación referida a la premisa de recibir a las parejas en mediación con la decisión de separarse ya tomada. Cuando empezamos a trabajar desde el servicio de mediación y nos encontrábamos ante parejas que no tenían cien por cien clara la decisión de separarse, nos planteábamos si podríamos ayudarles desde el espacio de mediación o sería más un trabajo de terapia. En nuestro interés prioritario de proteger a los menores, pensamos que en estos casos el espacio de mediación podría convertirse en un espacio preventivo de cara a la decisión de ruptura.

En la práctica diaria, son muchas las parejas que acuden al servicio de mediación sin una decisión firme. Es por ello que las mediadoras en nuestra labor de acompañamiento, recogemos y normalizamos las dudas, las inseguridades y los miedos que en muchas ocasiones acompañan a la decisión de la separación. Tal y como explicábamos antes, tomar la decisión de separarse no es sencillo, y pocas veces se tiene claro al cien por cien.

Con estas premisas, nos hacíamos la siguiente pregunta: ¿es posible mediar con parejas que no tienen clara la decisión de separase?

La respuesta, para nosotras, es sí. El espacio de mediación en estos casos, se convierte en un espacio de prevención en el que el mediador acompaña a la pareja a lo largo del difícil camino de tomar la decisión de separase y facilita herramientas para reflexionar y tomar las decisiones mas adecuadas para todos los miembros de la familia.

Volviendo a pensar en los menores, en nuestra experiencia, ayudar a los padres a ponerse en el lugar de sus hijos y transmitirles la idea de que los niños y niñas pueden ser inmensamente felices creciendo en una familia de padres separados.

Son muchas las parejas que mantienen la relación "por sus hijos/as". Temen que la ruptura les deje secuelas irreversibles y afecte a su desarrollo. La mediación en estos casos se plantea como un espa- 
cio en el que trabajar sobre las posibles consecuencias que el divorcio puede tener en los menores, pero también sobre las consecuencias que tiene para los niños/as crecer en un ambiente conflictivo. Las mediadoras, además ayudamos a la pareja a decidir el modo en que quieren transmitírselo a sus hijos, el modo en que quieren que comience a construirse su nuevo modelo de organización familiar. El mediador escucha, recoge la frustración y el dolor de la ruptura y les ayuda a convertirlo en un mensaje constructivo (orientado al futuro y no al pasado) que sus hijos puedan entender.

En la medida en que sea posible iniciar la mediación en esta primera etapa de la separación, mayor es la prevención del aumento del conflicto.

\section{¿Ya no somos pareja?}

Si tratamos de darle cierto orden cronológico al momento en el que desde la mediación se empieza a intervenir con una familia en situación de ruptura, lo siguiente que nos encontramos son parejas en proceso de divorcio, con muchas dificultades para afrontar los cambios (estructurales, emocionales, económicos, familiares) surgidos tras la decisión de la separación. Veíamos que tomar la decisión de separase, no es fácil, sin embargo es sólo la primera de muchas e importantes decisiones que los padres tendrán que tomar. Y es en este punto en el que situamos esta segunda tipología de familias.

Se trata de parejas inmersas ya en un proceso de divorcio (contencioso o amistoso), pero algo perdidas en las decisiones a tomar.

En este sentido, el espacio de mediación les ayuda a poner límites claros a los asuntos que tienen que ver con la conyugalidad y aquellos relacionados con la coparentalidad. Los motivos por los que la pareja se separa, o quién tomó la decisión, o si hubo infidelidades, son temas que corresponden al área de la relación de pareja y pasan a un segundo plano; sin embargo, cómo planteárselo a los niños/as o dónde van a vivir son decisiones que tienen que tomar como padres. Y este límite que las mediadoras nos esforzamos en señalar, es, en ocasiones difícil de entender y de poner en práctica. Entender cuando habla mi "yo-marido" y cuando lo hace mi "yo- padre", lleva tiempo y esfuerzo y además implica que hay un duelo que empezar a elaborar y una serie de cambios a los que enfrentarse.

El espacio de mediación se conforma como un espacio en el que aprender a separar la parte de pareja que se separa y la parte de padres que debe estar más unida que nunca. Se trata también de fortalecer y fomentar la coparentalidad. A menudo, planteamos a los padres la coparentalidad como un red que protege a sus hijos; si la red es poco resistente o tiene agujeros, los niños pueden colarse por esos agujeros y encontrarse perdidos, pero si la red es fuerte y firme, los menores se sentirán sujetos y contenidos.

No cabe duda, que hacer esta separación entre lo conyugal y lo parental supone una enorme dificultad ya que muchas veces se mezclan aspectos conyugales que interfieren en la práctica de la coparentalidad (siguiendo con la metáfora de la red, tendríamos una red llena de nudos difíciles de deshacer por los menores). También es habitual pasar al extremo contrario en el que la separación se hace extensible al área de la parentalidad, de forma que nos encontramos con padres absolutamente distantes en un intento de ser fieles a su decisión de separase (una red con tantos agujeros, que no puede sostener a los hijos/as).

En el espacio de mediación uno de los objetivos es resaltar la importancia de la coparentalidad y acompañar a las familias en la construcción de su nuevo modelo de relación parental.

El duelo de la separación tiene cabida en el espacio de mediación, en la medida en que interfiere en la construcción y/o fortalecimiento del espacio parental. Difícilmente los padres pueden construir su nueva relación de padres, si no se le deja un espacio al dolor que supone la pérdida.

\section{Cuando una sentencia no basta}

Si seguimos avanzando en el proceso de divorcio, la siguiente fase sería la legalización de la separación. Muchas familias esperan la sentencia como la "solución" a sus problemas. Cuando las parejas no han podido alcanzar un acuerdo, otorgan al juez el poder de decirles lo que tienen que hacer. A pesar de 
los años, sigue llamándonos la atención la forma en que las familias prefieren que sea un tercero el que tome las decisiones por ellos. Sin embargo, la experiencia nos muestra casi a diario que en muchas ocasiones las sentencias judiciales no sólo no resuelven la situación sino que a veces aportan confusión a la familia respecto al cumplimiento de la misma.

No son pocos los casos en los que nos hemos encontrado con parejas ya separadas o divorciadas en las que no se están cumpliendo los acuerdos o la sentencia. A veces, las sentencias no son tan concretas como las familias necesitan y dan lugar a diferentes interpretaciones que, a menudo terminan generando algún conflicto. Lo mismo ocurre con los procedimientos de mutuo acuerdo que, en ocasiones se complican a la hora de llevarlos a la práctica. El lenguaje del derecho, a veces no permite acercarse demasiado a las emociones de las familias en plena transformación.

Desde la práctica diaria, la pregunta que nos hicimos fue: ¿podemos mediar cuando hay ya una sentencia judicial o un acuerdo firmado? Desde una óptica reduccionista, podríamos preveer una respuesta negativa; si ya hay una sentencia, no hay más que hablar. Sin embargo desde "nuestro" espacio de mediación, respondemos a las necesidades de las familias, y si una de estas necesidades es aclarar qué quiere decir la sentencia en alguno o todos sus puntos, la mediación es una espacio para hacerlo.

Cada vez con mayor frecuencia, nos encontramos con familias que arrastran dificultades en su día a día que les generan conflictos, dolor, desencuentros, malentendidos, enfrentamientos. Todo esto va generando un clima de confusión y enfado que puede ser caldo de cultivo para un conflicto abierto que puede producirse en cualquier momento. Los menores viven inundados por este ambiente confuso que les genera incertidumbre.

En este sentido, el espacio de mediación se convierte en un espacio de reflexión en el que el mediador ayuda a las partes a concretar qué parte de los acuerdos no está funcionando (por ser ambiguos o demasiado teóricos) de una forma saludable, y facilita herramientas que les permitan adaptarse a la nueva situación y resolver posibles conflictos que puedan aparecer en el futuro. Pero sobre todo, devuelve el poder que estas personas habían deposi- tado en el contexto judicial, y los reconcilia con su propia capacidad de decidir lo más adecuado para sus familias.

De nuevo, el espacio se convierte en una herramienta con la que construir nuevos modelos de relación parental alternativos a los que no funcionan adecuadamente. Por un lado, esta forma de intervenir, hace que las familias se lleven su propio modelo creado en función de sus necesidades, pero además han podido introducir cambios en su relación basados en su propia capacidad de decidir y de asumir responsabilidades. Pero además, se llevan algo muy importante que es sentir que son ellos los que han decidido lo que es mejor para sus hijos/as.

\section{Más allá del acuerdo}

Una vez firmado el acuerdo, finaliza la mediación. $\mathrm{O}$ al menos así se plantea desde los diferentes modelos de mediación. Pero a lo largo de estos años de trabajo, en muchas ocasiones nos hemos planteado si realmente la firma del acuerdo marca el fin de la intervención.

Según nuestra experiencia, la firma del acuerdo es sólo la consecución de uno de los muchos objetivos que se pueden trabajar en mediación y es por eso que hemos sentido que el espacio de mediación podía seguir acompañando como seguimiento a la puesta en marcha del acuerdo.

En ocasiones, tras la firma del acuerdo, las familia se encuentra ante un abismo, un horizonte lleno de cambios y de situaciones nuevas a las que nunca se han enfrentado. Llevarse sus acuerdos en un papel, les ofrece cierta tranquilidad y satisfacción, pero no deja de ser teoría. Han sido varias las familias que tras la firma se/nos han preguntado si el trabajo había terminado. La primera vez que nos ocurrió, nos miramos las medidoras y dudamos en la respuesta. A partir de ese momento, cuando las familias nos han pedido continuar con la intervención, no hemos dudado en seguir adelante.

El espacio de mediación se configura de nuevo como acompañamiento. Poner en práctica los acuerdos alcanzados entraña alguna dificultad, miedo e incluso resistencias. Significa que, ahora sí, la separación es una realidad. Y esto, sin duda, asusta. Por 
eso cuando las familias lo solicitan, el espacio de mediación sigue activo para resolver cuestiones que puedan aparecen ante la puesta en marcha de los acuerdos. Se trataría de facilitar y acompañar en la difícil tarea de adaptarse al cambio familiar.

\section{No puedo verte}

Este quizá sea uno de los aspectos más llamativos de nuestra propuesta de intervención, al menos es lo que nos ha parecido cuando lo hemos comentado con otros profesionales. Sorpresa e incredulidad son algunas de las reacciones que nos hemos encontrado. Sin embargo, nosotras seguimos apostando por un modelo de mediación adaptado a las necesidades particulares de cada familia.

$\mathrm{Y}$ atendiendo a estas necesidades, en alguna ocasión nos hemos encontrado con lo que antes para nosotras era un impedimento; la petición explícita de la familia de no encontrarse en las sesiones. Cuando esto nos ha ocurrido, hemos tenido que finalizar el trabajo con estas parejas. Basándonos en los modelos más clásicos de mediación, veíamos esta petición como un impedimento para continuar trabajando en el espacio de mediación.

Sin embargo, nuestra sensación era de estar abandonando a las familias porque no se adaptaban al modelo de trabajo planteado. Y como esta sensación no nos gustaba en absoluto, nos hicimos la siguiente pregunta: ¿se puede hacer mediación con una pareja que pide explícitamente no verse? Imaginamos que la respuesta dependerá de a quién formulemos la pregunta, pero para nosotras, después de reflexionar mucho acerca de esta posibilidad, la respuesta en algunas ocasiones es si. No cabe duda, que uno de los objetivos del espacio de mediación es facilitar la comunicación entre las partes, pero qué ocurre cuando las partes no quieren verse, pero si negociar sobre su separación. Hablamos en concreto de parejas que inician la mediación, pero que cada sesión conjunta les supone un sufrimiento que les impide seguir adelante con el trabajo planteado. Sin embargo, tienen clara la importancia de alcanzar un acuerdo y de seguir negociando. En estos casos nos centramos en resaltar la capacidad negociadora a las familias y no tanto en seguir un protocolo de actua- ción rígido que a veces impide que las familias puedan alcanzar soluciones adecuadas.

De nuevo el espacio de mediación se flexibiliza y prioriza aquella parte de las familias que sí les permite trabajar para conseguir objetivos, y minimiza la parte formal que a priori podría verse como un obstáculo o incluso como un impedimento.

Nos gustaría dejar claro que la posibilidad de trabajar en mediación sin recibir a las partes de manera conjunta, no es una posibilidad que las mediadoras planteemos a las familias. No lo es, porque seguimos pensando que el espacio de mediación lo constituyen las partes, las familias. Trabajar y negociar por separado es sólo un "experimento" que nos ha dado buen resultado.

\section{Porque lo dice el juez}

Desde hace cinco años, los Centros de Apoyo a las Familias en coordinación con los Juzgados de Familia de Madrid, vienen proporcionando un punto de información sobre mediación (PIM) a aquellas familias que los jueces deciden derivar. Se trata de familias inmersas en un procedimiento judicial contencioso. El criterio por el que los jueces y juezas derivan a las familias, sólo lo conocen ellos, pero se trata sin duda de una oportunidad que los magistrados conceden a las familias antes de tener que decidir por ellos.

¿Entraría en cuestionamiento el principio de la voluntariedad tantas veces hablado?. En nuestra opinión, las parejas acuden a ser informadas sobre mediación por mandato judicial pero en el momento en que se les explica en qué consiste, se les recuerda la voluntariedad del proceso. Las familias no están obligadas a iniciar mediación, simplemente el juez o jueza ha considerado que podría ser beneficioso para ellos y, sobre todo, para sus hijos e hijas.

¿Se puede trabajar en mediación con una familia que tiene uno o varios procedimientos judiciales abiertos? A todos los que nos hemos formado en mediación, se nos ha transmitido siempre la incompatibilidad de ambos procedimientos. Y, ciertamente resulta, a priori, algo incompatible. Bajo nuestro punto de vista, no tiene mucho sentido estar peleando en los juzgados mientras se está negociando en el 
espacio de mediación. Sin embargo, la mediación (siempre flexible) se adapta a los tiempos que marcan los procedimientos judiciales; es decir, aprovechamos los tiempos que hay entre las vistas para trabajar en mediación, de forma que no sea necesario suspender el procedimiento (salvo que ambas partes así lo decidan).

Una vez que la familia acepta beneficiarse del espacio de mediación, empezamos a trabajar con ellos con un primer y fundamental objetivo en la cabeza: devolver a las familias la capacidad de resolver y tomar decisiones acerca de si mismos. Recordemos que cuando las familias inician procedimientos contenciosos, automáticamente pasan a sentir que no pueden tomar decisiones y que éstas, dependen de lo que el juez decida. Por nuestra experiencia, las parejas que acuden a una primera sesión de información de mediación, procedentes del juzgado, en ocasiones no son conscientes de sus propias capacidades de resolución, de comunicación y de toma de decisiones. No seríamos honestas si no dijéramos que la dificultad a la hora de trabajar con estas familias es mucho mayor que con familias que voluntariamente deciden acudir a mediación. Se trata de casos con un altísimo nivel de conflicto, que, en muchos casos llevan años depositando la capacidad de decidir en un tercero (el juez) y confiando en que sea éste el que les marque el camino.

En el espacio de mediación les invitamos a que sean ellos los que tomen la palabra que en un momento decidieron darle al juez. En estos casos, el mediador se encuentra con familias con una comunicación que suele estar muy deteriorada y con escasa confianza en sus propias capacidades y en el contexto mediador. Es difícil que viniendo de un espacio de confrontación, y acompañados por profesionales con un perfil tan diferente al del mediador, puedan sentir este espacio como una herramienta terapéutica que les aporte soluciones en lo práctico. En estos casos, el acompañamiento del mediador consiste en hacer del espacio de mediación un lugar confiable y cercano en el que son ellos quienes deciden, algo que quizá por estar inmersos en un procedimiento judicial, no tenían tan claro. Nos planteamos un objetivo fundamental a la vez que complicado de poner en práctica; que ambos padre sientan que la mediación está de su parte. Aparcamos la lógica de que sólo uno puede tener razón, la lógica de "si una gana el otro pierde". Les animamos a hablar, a escucharse, a mirarse, a contarse cosas de sus hijos. Y sólo cuando el espacio dé confianza, se puede empezar a pensar en proponer y alcanzar acuerdos.

\section{Los hijos también cuentan}

Plantear la inclusión de los menores en el espacio de mediación es una cuestión muy debatida por los profesionales que trabajamos con familias. Por un lado, si nos guiamos por los modelos más clásicos de mediación, dar un espacio a los menores dentro del proceso de mediación no tendría sentido, puesto que se trata de hacer justo lo contrario, mantener a los hijos al margen y devolver a los padres la responsabilidad de resolver aquello que les está impidiendo funcionar adecuadamente y que además está provocando malestar en sus hijos.

Sin embargo, y con esto volvemos a las premisas de las que hablábamos al inicio de este artículo, con el paso de los años, en muchas ocasiones nos hemos planteado la posibilidad de ofrecer un espacio a los hijos por varios motivos. En primer lugar, estamos convencidas que ante un divorcio mal resuelto, los grandes perdedores con los menores, y por eso sentimos que en algunos casos merecen tener un espacio de escucha. Pero además creemos que los hijos pueden movilizar mucho más de lo que lo hace el propio espacio de mediación.

Por otra parte, creemos que antes de poder sacar algún elemento del sistema (familia), es necesario incluirlo primero, es decir, el objetivo de recibir a los menores no es otro que poder excluirlos del espacio despojándolos de cualquier responsabilidad relacionada con la ruptura. De esta forma, lograríamos transmitir a los hijos e hijas que estamos ayudando a sus padres a resolver la situación y a decidir lo mejor para ellos. Se trata de un mensaje de tranquilidad y de futuro; "mis padres se están trabajando juntos para ofrecerme lo mejor”. Según nuestra experiencia, en la medida en que los hijos e hijas reciben la información consensuada de que los padres están trabajando juntos para decidir qué es lo mejor para ellos, los menores lo reciben como un 
mensaje de protección y sienten que sus padres están siendo capaces de ponerse en su lugar.

Los niños cuyos padres están resolviendo adecuadamente su separación, puede dedicar su energía a jugar, a aprender, a estudiar, a crecer. Por el contrario, aquellos menores que se encuentran atrapados entre sus padres, no pueden centrarse en sus asuntos y empiezan a presentar sintomatología que, si no se detiene a tiempo, puede derivar en problemas más graves como disminución del rendimiento escolar, falta de concentración, baja autoestima, inseguridad, dificultades en las relaciones con los iguales, depresión, apatía.

Es por esto, que las mediadoras muchas veces nos presentamos ante los padres como las portavoces de sus hijos. Y tratamos de que, ante cada decisión, confrontación, duda, miedo, amenaza, pregunta, etc., los padres puedan ponerse en el lugar de sus hijos y preguntarse qué sería lo mejor para ellos. De esta forma, los padres reciben un mensaje importante: pueden y deben tener en cuenta las opiniones y deseos de sus hijos e hijas, pero la toma de decisiones les corresponde a ellos.

Con estas siete categorías, hemos querido reflejar la diversidad de familias y necesidades con la que nos encontramos día a día. Seguramente, con el paso de los años, nos encontraremos con nuevas demandas y nuevas formas de construir el espacio de mediación desde la flexibilidad y el deseo de poder acompañar a las familias en los momentos de cambio.

\section{El acompañamiento de los mediadores al resto de profesionales implicados en el contexto de mediación. Un contexto de mediación más amplio}

Otra parte importante que debemos tener en cuenta dentro del espacio de mediación es la coordinación con los diferentes profesionales que intervienen con las familias. Sabemos la importancia que tiene que todos los profesionales que estamos interviniendo con familias, llevemos una línea de actuación coherente y en sintonía. Por eso, cuando arrancó el servicio de mediación de los Centros de Apoyo a las Familias, dedicamos parte de nuestros esfuerzos a divulgarlo por la red de servicios de ayuda a las familias, tratando de transmitir a numerosos profesionales, de que manera podemos ayudar en mediación para facilitar la derivación a nuestros centros.

Además sabemos la especial relevancia que tiene para algunas familias que empiezan a trabajar en mediación, el papel de abogados, jueces, equipos psicosociales. Incluir a estos profesionales en el espacio de mediación, es importante para nosotras, y sobre todo para las familias. Contar con su colaboración significa enriquecer el espacio de mediación y devolver a la familia un mensaje de unidad y de apoyo a su proceso. Esto, quizá pueda chocar con el planteamiento clásico de mediación desde el que el espacio para otros profesionales está más limitado. Desde nuestra "construcción" de un nuevo espacio de mediación, nos gusta pensar y transmitir a las familias que otros profesionales (que son importantes para ellos) tienen cabida. En nuestra experiencia, esto ofrece tranquilidad a las familias, y en muchas ocasiones le hace ver que no es necesario elegir, que los abogados o los jueces pueden estar presentes en el espacio de mediación ${ }^{2}$.

Por otro lado, creemos importante reforzar no sólo el trabajo de coordinación sino también la divulgación del espacio de mediación de cara a poder hacernos un hueco estable y confiable en el sector de la intervención con familias. No olvidemos que la mediación es todavía novedosa para muchos profesionales y eso implica que hemos de darnos a conocer y no esperar a que nos presenten sino a presentarnos a nosotros mismos y no tener miedo a cansarnos y seguir haciéndolo durante el tiempo que sea necesario.

Además, entendemos que la definición de mediación que puede estar llegando a otros sectores en una concepción clásica de mediación en la que ésta se define como un contexto protagonizado única y exclusivamente por "el mediador y las partes", siendo el resto de profesionales, ajenos a la mediación, y a sus resultados.

En este sentido, nuestra propuesta desde el acompañamiento en que se basa este modelo, es acompa-

Hablamos de una presencia diferente, desde otro lugar desde el que poder asesorar a sus clientes, pero dejándoles a ellos tomar protagonismo en la toma de decisiones. 
ñar también al resto de profesionales que están implicados en este campo desde la divulgación clara de cuál es nuestro papel y nuestro trabajo. Nosotras creemos que una mediación eficaz es aquella que pasa por una transmisión clara a otros profesionales de cómo es nuestra intervención, eso contribuiría a generar y definir una nueva relación con los profesionales, algo que ahora es bastante difuso.

Nuestra apuesta pasaría por un cambio de punto de vista. De esta forma, definiríamos el contexto de mediación, "grosso modo" como un sistema que integra a muchos más participantes y a intereses muy variados, más amplio que el clásico, el mediador y las partes, más variado, más completo y complejo; que aglutina a muchos más profesionales: los abogados, los trabajadores sociales, la familia extensa, los jueces, los equipos psicosociales; ya que todos ellos están implicados en esa búsqueda de consenso, de facilidad y de acompañamiento en la toma de decisiones en momentos de cambio.

Creemos que desde esa visión del contexto de mediación integradora y compleja, y desde una posición proactiva de los mediadores hacia otros profesionales implicados en el contexto de ayuda a las familias, haremos posible un mejor trabajo a todos los niveles, nos conocerán y conoceremos, evolucionaremos todos, familias y profesionales en los contextos de cambio.

\section{Reflexiones finales}

Con todo lo planteado hasta aquí, con la múltiples reflexiones que nos ha llevado la elaboración de este artículo y con la imprescindible colaboración de nuestros compañeros mediadores de los diferentes CAF, y de nuestro compañero supervisor Ignacio Bolaños, nos hemos atrevido a definir la mediación como un "espacio flexible de comunicación donde sus protagonistas, acompañados de una persona mediadora, toman las decisiones necesarias para facilitar la situación derivada del cambio familiar en el que están inmersas".

Esta definición nos permite trabajar en mediación con una casuística amplia sin limitaciones de tipologías que en cierto sentido no permitían abordar toda la amplia gama de dificultades por las que puede atravesar una familia. Y gracias a ella, nuestro trabajo hoy en el espacio de mediación es mucho más rico y nos permite mirar y acompañar a las familias desde un espacio mas amplio, que, según nuestra experiencia, engrandece y potencia las posibilidades del espacio de mediación.

\section{Referencias}

Bernal Samper, Trinidad. (2002) La mediación: una solución a los conflictos ruptura de pareja. Madrid: Colex.

Bolaños Cartujo, Ignacio. (2008). Hijos alineados y padres alienados. Mediación familiar en rupturas conflictivas. Madrid: Reus.

Haynes, John M.(1995). Fundamentos de la mediación familiar. Madrid: Plaza edición.

Maganto Mateo, Carmen (2004). Mediación familiar: aspectos psicológicos y sociales. San Sebastián.

Manuscrito recibido: 07/01/2011

Revisión recibida: 17/03/2011

Aceptado: 21/03/2011 